\title{
Assessing the impact of PCSK9 inhibition on coronary plaque phenotype with optical coherence tomography: rationale and design of the randomized, placebo-controlled HUYGENS study
}

\author{
Stephen J. Nicholls, Steven E. Nissen, Francesco Prati, Stephan Windecker, Yu Kataoka, Rishi Puri, \\ Thomas Hucko, Helina Kassahun, Jason Liao, Ransi Somaratne, Julie Butters, Giuseppe Di Giovanni, \\ Stephen Jones, Peter J. Psaltis \\ Monash Cardiovascular Research Centre, Clayton, Australia \\ Contributions: (I) Conception and design: All authors; (II) Administrative support: All authors; (III) Provision of study materials or patients: All \\ authors; (IV) Collection and assembly of data: All authors; (V) Data analysis and interpretation: All authors; (VI) Manuscript writing: All authors; (VII) \\ Final approval of manuscript: All authors. \\ Correspondence to: Stephen J. Nicholls. Monash Cardiovascular Research Centre, 246 Clayton Road, Clayton, VIC 3168, Australia. \\ Email: stephen.nicholls@monash.edu.
}

Background: Technological advances in arterial wall imaging permit the opportunity to visualize
coronary atherosclerotic plaque with sufficient resolution to characterize both its burden and compositional
phenotype. These modalities have been used extensively in clinical trials to evaluate the impact of lipid
lowering therapies on serial changes in disease burden. While the findings have unequivocally established
that these interventions have the capacity to either slow disease progression or promote plaque regression,
depending on the degree of lipid lowering achieved, their impact on plaque phenotype is less certain. More
recently optical coherence tomography (OCT) has been employed with a number of studies demonstrating
favorable effects on both fibrous cap thickness (FCT) and the size of lipid pools within plaque in response to
statin treatment.

Methods: The phase 3, multi-center, double-blind HUYGENS study will assess the impact of incremental lipid lowering with the proprotein convertase subtilisin kexin type 9 (PCSK9) inhibitor, evolocumab, on plaque features using serial OCT imaging, in statin-treated patients following an acute coronary syndrome (ACS). Subjects with non-ST-elevation ACS ( $\mathrm{n}=150)$ will be randomized 1:1 into two groups to receive monthly injections of evolocumab $420 \mathrm{mg}$ or placebo.

Results: The primary endpoint is the effect of evolocumab on coronary atherosclerotic plaques will be assessed by OCT at baseline and at week 50 .

Conclusions: The HUYGENS study will determine whether intensified lipid lowering therapy with evolocumab in addition to maximally tolerated statin therapy will have incremental benefits on high-risk features of coronary artery plaques.

Trial registration: This study was registered on Clinicaltrials.gov (NCT03570697).

Keywords: Imaging; lipids; clinical trials; proprotein convertase subtilisin kexin type 9 (PCSK9); risk factors

Submitted Aug 03, 2020. Accepted for publication Dec 29, 2020.

doi: $10.21037 / \mathrm{cdt}-20-684$

View this article at: http://dx.doi.org/10.21037/cdt-20-684 


\section{Introduction}

Randomized controlled trials have unequivocally established that lowering levels of low-density lipoprotein cholesterol (LDL-C) reduces cardiovascular event rates in both the primary and secondary prevention setting (1). Meta-analyses have subsequently demonstrated a direct association between the degree of LDL-C reduction and cardiovascular benefit (1). As a result, treatment guidelines for cardiovascular prevention have increasingly emphasized the use of intensive statin therapy for higher risk patients (2). However, the observation of a considerable residual clinical risk in statin-treated patients related to insufficient LDL-C targets $(3,4)$. And the emerging reality of statin intolerance has supported the need to develop additional LDL-C lowering strategies for use in clinical practice (5).

\section{Lipid lowering beyond statins}

A number of therapeutic agents have been developed for patients who are not able to achieve satisfactory LDL-C lowering despite use of maximally tolerated statin therapy. Ezetimibe is a cholesterol absorption inhibitor, which lowers LDL-C by $15-25 \%$ when administered as monotherapy or in addition to statin therapy (6). Two large clinical outcomes trials have demonstrated that ezetimibe administration on top of statin therapy results in a reduction in cardiovascular events in patients with end stage kidney disease and on long term follow up after an acute coronary syndrome (ACS) $(7,8)$. Bempedoic acid inhibits ATP citrate lyase, a factor which similarly to statins is involved in the hepatic cholesterol synthesis pathway $(9,10)$. As a result, this agent has the ability to lower LDL-C levels by $16-25 \%(11,12)$. The effect of this agent on cardiovascular outcomes is currently being evaluated in a large clinical trial of high-risk patients with statin intolerance and hypercholesterolemia.

Proprotein convertase subtilisin kexin type 9 (PCSK9) inhibitors present the opportunity to profoundly lower LDL-C. Genetic studies associate gain of function PCSK9 mutations with hypercholesterolemia (13) and loss of function polymorphisms with both low LDL-C levels and a lower prevalence of cardiovascular disease $(14,15)$. Subsequent genetic and biochemical studies have established that PCSK9 plays an important role in the regulation of cholesterol metabolism, by lowering expression of the LDL receptor on the hepatocyte surface $(13,16,17)$. Development of inhibitory monoclonal antibodies have been demonstrated to effectively reduce PCSK9 activity and to lower LDL-C in a dose dependent fashion on average to $60 \%$ when administered as monotherapy or in combination with statin therapy (18-20). This provides the opportunity to achieve effective lipid lowering, particularly in patients with statin intolerance and familial hypercholesterolemia, but also to reduce LDL-C to very low levels in those patients already treated with a statin $(21,22)$. Two large outcomes trials have demonstrated that lowering LDL-C to much lower levels than previously observed reduced cardiovascular events in patients with either stable atherosclerotic cardiovascular disease (23) or in those with an ACS in the prior 4-52 weeks (24). It is therefore likely that the patient at elevated cardiovascular risk warrants efforts to lower LDL-C to very low levels.

\section{Effect of statins on atherosclerotic plaque using arterial wall imaging}

Technological advances in arterial wall imaging have produced a range of modalities with the ability to not only localize plaque in a range of vascular beds, but also to quantify its burden and characterize its composition. Early studies demonstrating the benefit of statins on disease progression using coronary angiography $(25,26)$ and carotid intima-medial thickness $(27,28)$ have been extended to intravascular ultrasound (IVUS), which examines the full burden of coronary atherosclerosis. Clinical trials employing serial IVUS imaging have demonstrated that increasingly intensive statin therapy slows disease progression and promotes plaque regression, with the degree of benefit proportional to the extent of LDL-C lowering achieved (29-31). Additional studies have revealed that C-reactive protein (CRP) lowering with statins independently associates with their impact on plaque progression, supporting reports that statins possess antiinflammatory properties $(32,33)$.

With increasing interest in the potential for plaque composition to be an important determinant of the translation of atherosclerotic disease to clinical manifestation, considerable attempts have been made to characterize the effects of statins on plaque morphology, beyond its size. Pathology studies have demonstrated that statin treatment prior to endarterectomy produces atheroma that contains less lipid and inflammatory material, supporting a potential role for plaque stabilization (34). While IVUS imaging lacks the resolution to effectively visualize plaque components, serial studies have reported 
that statin use associates with progressive plaque calcification (35). Studies using different imaging modalities have reported that statins may also reduce plaque lipid on near infrared spectroscopy (NIRS) (36), necrotic core on magnetic resonance imaging (MRI) (37) and the degree of inflammatory activity using positron emission tomography (PET) (38). While these findings align with the observations from pathology studies, these modalities have been less commonly used in serial imaging studies of antiatherosclerotic therapies.

Intracoronary optical coherence tomography (OCT) is a catheter-based imaging technique using coherent near infrared light to generate images with micrometer spatial resolution from optical backscatter, thus enabling detailed imaging of the intimal aspects of the coronary artery wall. As a result, OCT has been established to visualize the fibrous cap and underlying lipid pools with high resolution, permitting their measurement in vivo. Numerous reports have emerged from OCT-based studies demonstrating the presence of hallmark features of vulnerable plaque, including a thin fibrous cap, large lipid pool, cholesterol crystals, spotty calcification, neovascularization and potentially macrophage collections in culprit lesions (39-42) and other sites within the vasculature $(43,44)$ of ACS patients. Furthermore, the presence of a lipid rich plaque, as evidenced primarily by a thin fibrous cap, but also with wide lipid arc and presence of macrophages on OCT, has been reported to associate with a greater rate of cardiovascular events on long term follow-up (45).

This technique has also been employed to study the effects of LDL-C levels and statins on atherosclerotic plaque. Observational studies have demonstrated that higher LDL-C levels associate with a thinner fibrous cap and large lipid pool (46), while use of more intensive statin therapy associates with a greater fibrous cap thickness (FCT) and less evidence of neovascularization (47). Clinical trials using serial OCT imaging have demonstrated that statin therapy has a favorable effect on plaque, as evidenced by an increase in FCT and a reduction in the size of the lipid pool (47-53). These findings provide an important contribution to the literature understanding the mechanistic effects of statins on plaque.

\section{Effect of the PCSK9 inbibitor, evolocumab, on atherosclerotic plaque using arterial wall imaging}

In parallel with the outcomes trials, the effect of the PCSK9 inhibitor, evolocumab, on coronary atherosclerosis has been studied in the Global Assessment of Plaque Regression with a PCSK9 Antibody as Measured by Intravascular Ultrasound (GLAGOV) study. This study compared the effects of treatment with evolocumab or placebo on plaque progression in statin-treated patients with angiographic coronary artery disease (CAD). Addition of evolocumab reduced LDL-C from 93.0 to $36.6 \mathrm{mg} / \mathrm{dL}$, and was associated with a reduction in percent atheroma volume (PAV) by $0.95 \%$ and a greater proportion of patients demonstrating any degree of plaque regression over 18 months (54).

A virtual histology substudy reported progressive increases in plaque calcium with both statin monotherapy and when used in combination with evolocumab. The inverse association between achieved LDL-C levels and plaque calcification, suggests that this effect is a result of lipid lowering, rather than a pleiotropic effect of statins (55). A study in 129 subjects assessed the effect of evolocumab compared to placebo on arterial wall inflammation, in patients with elevated $\mathrm{Lp}(\mathrm{a})$ but did not meet the primary endpoint, as measured by fluorodeoxyglucose (FDG) PET uptake (56). Whether this reflects a lack of impact of evolocumab on plaque inflammatory activity, resistance to modification in the setting of elevated $\mathrm{Lp}$ (a) levels or a limitation of FDG scanning as a tool to evaluate medical therapies is uncertain. Nevertheless, there is an ongoing interest to further characterize the effects of PCSK9 inhibition on atherosclerotic plaque.

\section{HUYGENS study objective}

The High-Resolution Assessment of Coronary Plaques in a Global Evolocumab Randomized Study (HUYGENS) will employ serial OCT imaging to evaluate the impact of PCSK9 inhibition with evolocumab on coronary atheroma phenotype. The primary objective is to evaluate the effect of evolocumab on changes in FCT in patients with a non-ST-elevation ACS taking maximally tolerated statin therapy. Additional objectives are to evaluate the impact of evolocumab on other measures of plaque phenotype and the safety and tolerability of evolocumab when administered to patients in the post ACS setting. The primary hypothesis of the study is that achieving a low LDL-C with evolocumab in combination with statin therapy will result in a greater increase in the minimum FCT compared with statin monotherapy. We present the protocol in accordance with the SPIRIT reporting checklist (available at http://dx.doi. org/10.21037/cdt-20-684). 
150 patients with (i) NSTEMI, (ii) angiographic CAD, (iii) LDL-C $\geq 60 \mathrm{mg} / \mathrm{dL}$ on high-intensity, $\geq 80 \mathrm{mg} / \mathrm{dL}$ on low/ moderate-intensity or $\geq 130 \mathrm{mg} / \mathrm{dL}$ on no statin at screening, (iv) subsequently treated with maximally tolerated statin and $(v)$ target segment on OCT containing at least one image with a FCT $<120 \mu \mathrm{m}$ and one image with lipid arc $>90^{\circ}$

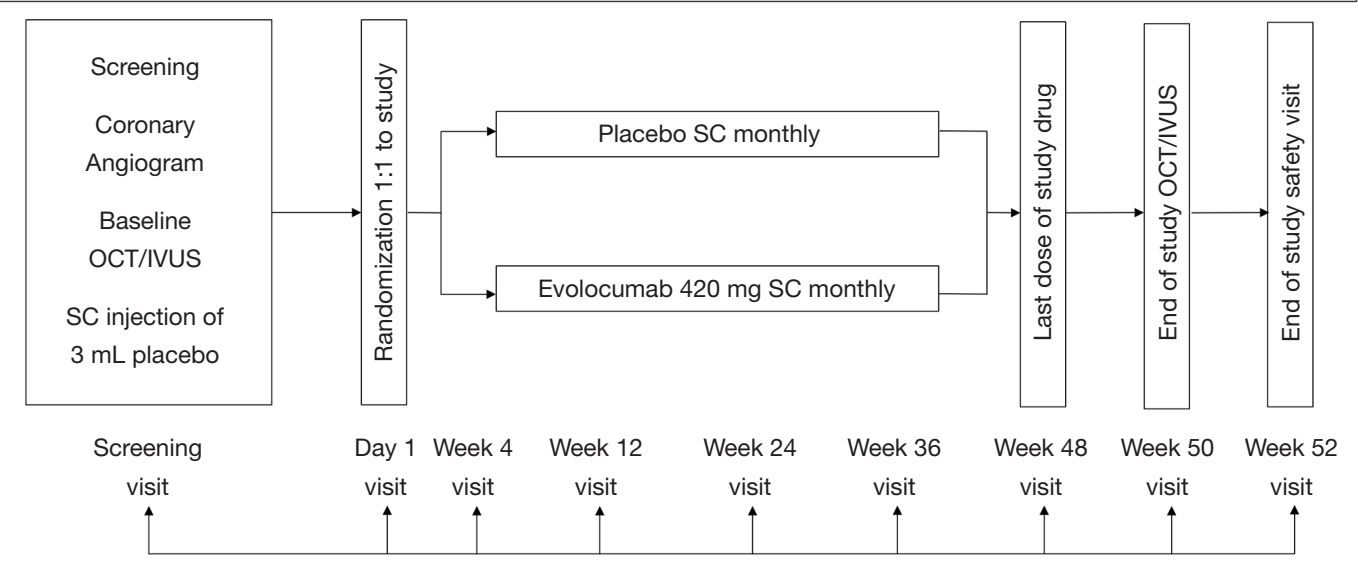

Figure 1 HUYGENS study design. NSTEMI, non-ST-elevation myocardial infarction; CAD, coronary artery disease; LDL-C, low-density lipoprotein cholesterol; FCT, fibrous cap thickness; IVUS, intravascular ultrasound; OCT, optical coherence tomography; SC, subcutaneous.

\section{Methods}

All procedures in this study will be conducted in accordance with the Declaration of Helsinki (as revised in 2013), International Conference on Harmonization, Good Clinical Practice guidelines, and applicable regulatory requirements. The final protocol was reviewed and approved by Ethics Committees and Institutional Review Boards at each study site and by Amgen Inc. (No. RES-18-0000-283A). All study participants will provide informed consent before participating in this study.

\section{HUYGENS study design and inclusion criteria}

HUYGENS (ClinicalTrials.gov Identifier: NCT03570697) is an ongoing (study commenced November 2018) randomized, double-blind, placebo-controlled, global, clinical trial to evaluate the impact of evolocumab on plaque phenotype. Eligible patients will include those who are (I) at least 18 years of age, (II) able to provide written, informed consent, (III) undergoing clinically indicated coronary angiography during admission due to non-STsegment elevation ACS with interventional treatment of culprit plaque, (IV) have a qualifying LDL-C level at the time of screening depending on their use of either no statin (LDL-C $\geq 130 \mathrm{mg} / \mathrm{dL}$ ), low or moderate intensity statin (LDL-C $\geq 80 \mathrm{mg} / \mathrm{dL}$ ) or high intensity statin (LDL-C $\geq 60 \mathrm{mg} / \mathrm{dL}$ ) and $(\mathrm{V})$ on maximally tolerated statin therapy in accordance with standard of care per local guidelines prior to randomization. In addition, patients must have evidence of (I) an angiographic stenosis of $\geq 20 \%$ in addition to the culprit plaque, (II) no left main coronary artery stenosis $>50 \%$, (III) a target vessel for imaging which cannot be deemed to be the culprit artery for the index or prior MI, has not undergone or intended to undergo revascularization and must be accessible by an OCT imaging catheter and (IV) an arterial segment containing no stenosis $>50 \%$, be at least $40-\mathrm{mm}$ in length and containing at least one image with a FCT $\leq 120 \mu \mathrm{m}$ and one image with a lipid arc $>90^{\circ}$. Exclusion criteria for the study include the presence of an ST-elevation MI, triglyceride levels $\geq 400 \mathrm{mg} / \mathrm{dL}$, moderate to severe renal dysfunction (eGFR $<30 \mathrm{~mL} / \mathrm{min} / 1.73 \mathrm{~m}^{2}$ ), malignancy, statin intolerance, prior or current use of PCSK9 inhibitors, women who are pregnant or breastfeeding or intend to become pregnant and any other condition deemed by the treating physician to impair the ability of the patient to comply with all study related procedures. All patients who meet all of the inclusion criteria, none of the exclusion criteria and who tolerate the placebo run-in injection at the time of screening will be deemed eligible and proceed to randomization within 1 week of providing informed consent.

Patients will be randomized to treatment with evolocumab $420 \mathrm{mg}$ or matching placebo administered by subcutaneous (SC) injection monthly for 48 weeks (Figure 1). Randomization will be stratified by current statin use 

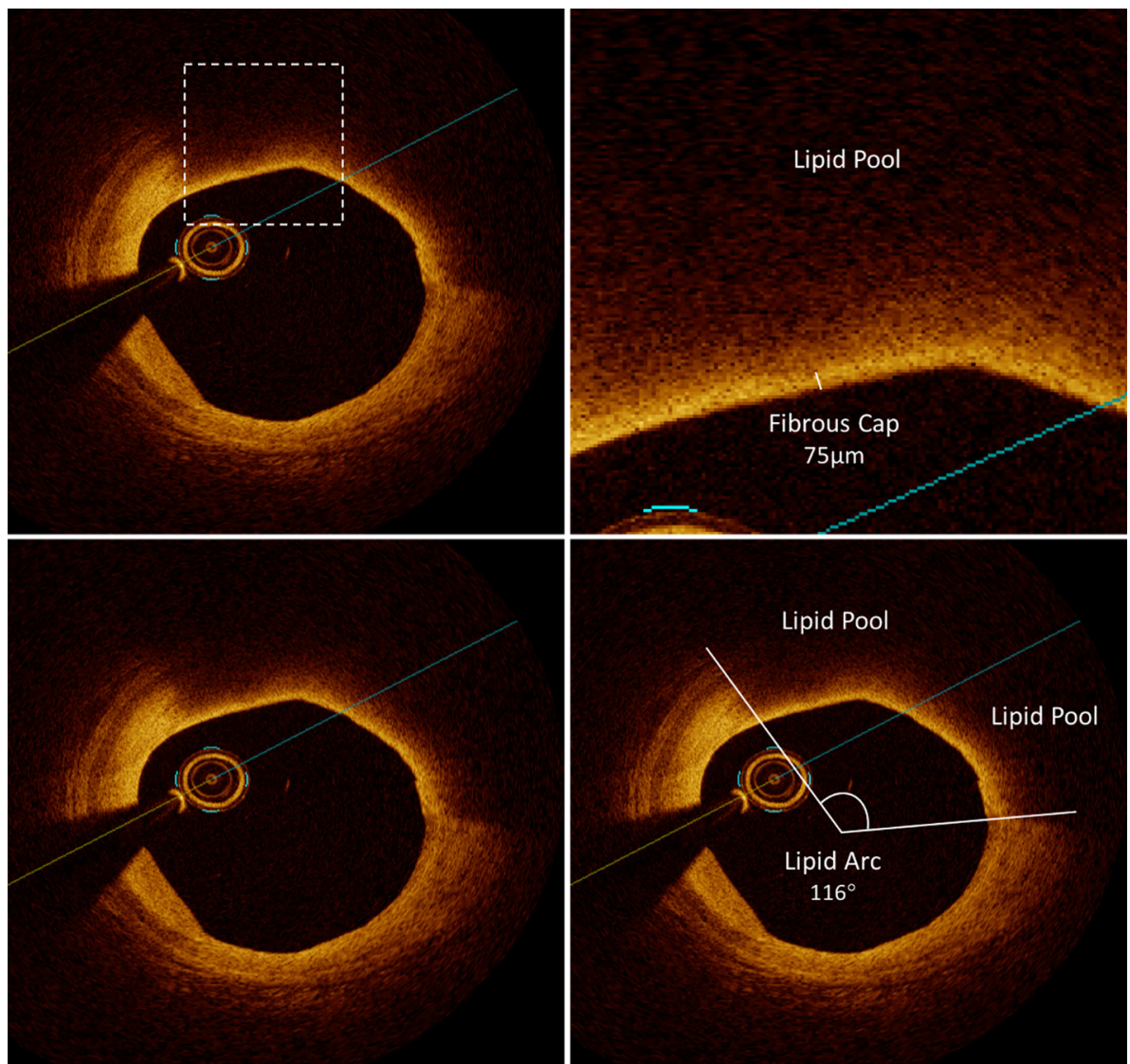

Figure 2 OCT plaque measurements. Cross-sectional plaque images acquired with OCT and demonstration of measurements of FCT (upper panels) and lipid arc (lower panels). OCT, optical coherence tomography; FCT, fibrous cap thickness.

( $>4$ or $\leq 4$ weeks duration) at screening. Study visits will be performed in the clinic at weeks 4 and 24 and by telephone at weeks 12 and 36. Imaging of the target artery by OCT and IVUS will be performed at the time of the screening visit coronary angiography and then repeated at week 50. A final end-of-study telephone call will be performed at week 52 to complete the safety evaluation.

\section{Image acquisition and analysis}

Intravascular imaging with OCT and IVUS will be performed within the target artery selected for investigation at both baseline and end of study. OCT imaging will be acquired by placement of an OCT imaging catheter (DragonFly Optis: Abbott) as distally as possible and withdrawn to the aorta by automatic pullback at a speed of $36 \mathrm{~mm} / \mathrm{second}$. The IVUS imaging will be acquired in a similar fashion, using an IVUS imaging catheter (OptiCross 40 MHz: Boston Scientific; Revolution 45 MHz: Phillips) at an automatic pullback speed of $0.5 \mathrm{~mm} /$ second. All imaging performed will be electronically transferred to the central core laboratory at the South Australian Health and Medical Research Institute. Screening OCT imaging will be assessed for image quality and to determine that the patient meets all eligible imaging inclusion criteria. Once a patient has completed the study and has evaluable imaging at both time points, measurements of plaque phenotype and burden will be performed. The segment selected for analysis will be defined by proximal and distal side branches and by the presence of at least one image containing a FCT $\leq 120 \mu \mathrm{m}$ and one image with a lipid $\operatorname{arc}>90^{\circ}$. The same segment will undergo measurements of both OCT and IVUS imaging by analysts who are blinded to the treatment status of the patient and to imaging timepoint (baseline/follow-up). For the OCT imaging, cross-sectional images, spaced $0.2 \mathrm{~mm}$ apart will be selected for analysis. For each image, where plaque is present, measurements of minimum FCT and the lipid arc will be performed (Figure 2). Each image will 


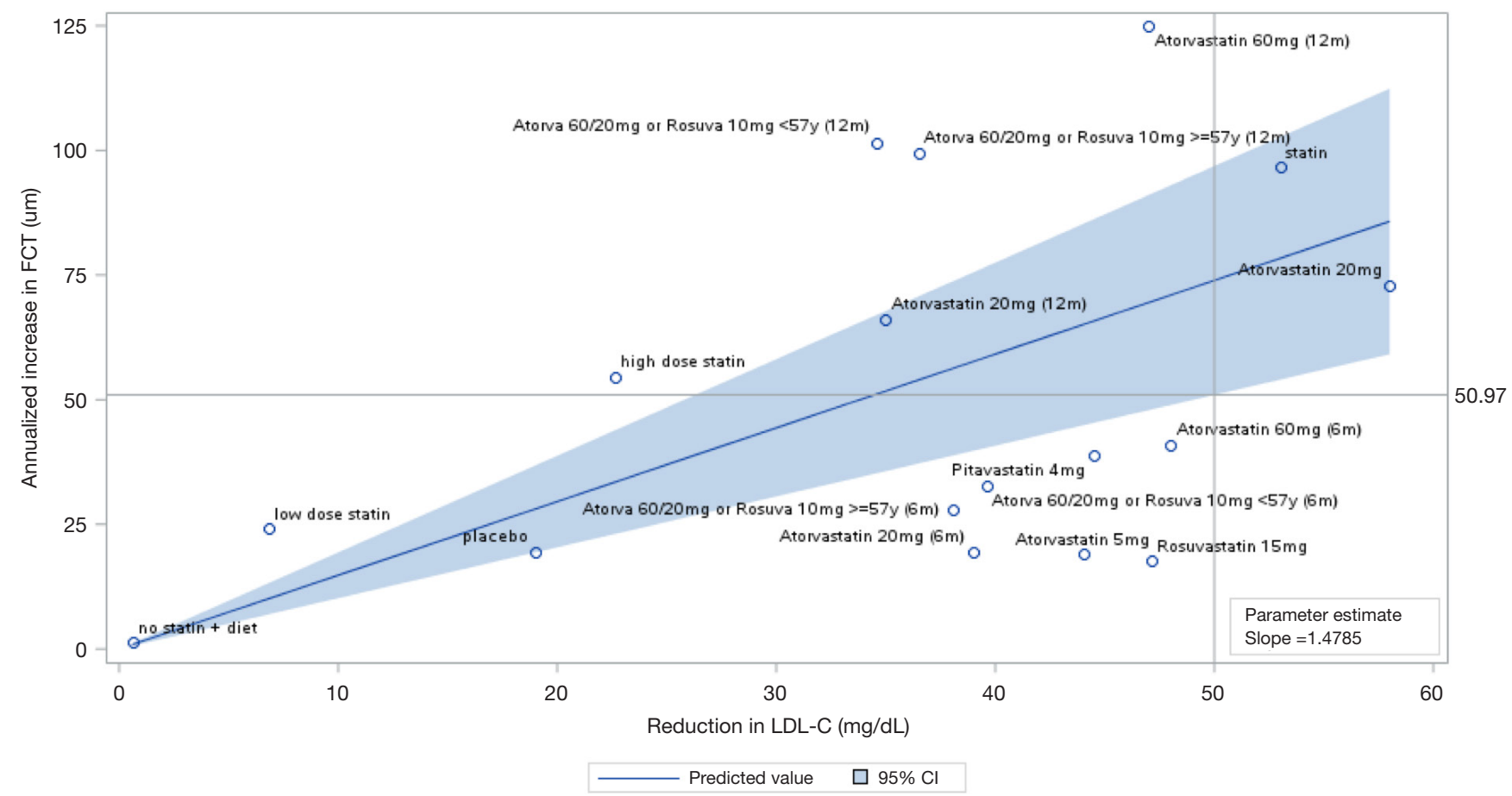

Figure 3 LDL-C reduction and change in FCT. Relationship between reduction in LDL-C and annualized change in FCT in trials that have employed serial OCT imaging to evaluate the effect of statin therapy. Given the invasive nature of the imaging performed in this study, it is assumed that $16 \%$ of patients will not have evaluable imaging at both time points. Accordingly, 150 patients or more will be randomized 1:1 to treatment with evolocumab $420 \mathrm{mg}$ or placebo to ensure there will be at least 125 patients completing the study. LDL-C, low-density lipoprotein cholesterol; FCT, fibrous cap thickness; OCT, optical coherence tomography.

be graded for the presence of macrophage and calcium accumulation (40). For IVUS imaging, cross-sectional images, spaced $0.5 \mathrm{~mm}$ apart, will be selected for analysis. The leading edges of the external elastic membrane (EEM) and lumen will be defined by manual planimetry. Plaque area in each image will be calculated as the area between the EEM and lumen. PAV will be calculated as the average of the proportion of plaque to EEM area in all images. Total atheroma volume (TAV) will be calculated as the average plaque area and normalized by the median number of images in the cohort. Regression will be defined as any decrease in PAV or TAV from baseline.

\section{Statistical analysis}

The primary and secondary endpoints will be analyzed by analysis of covariance (ANCOVA) with the covariates of treatment group, patient demographics, tobacco usage, type 2 diabetes mellitus, stratification factor of statin use at screening, baseline LDL-C, and baseline FCT. For missing post-baseline FCT measurements, values to be used for the primary and secondary endpoints will be imputed by multiple imputation. In addition, non-parametric Quade test will be used for the sensitivity analysis on the primary endpoint.

The assumptions in the sample size calculation are based on a linear regression meta-analysis of seven studies that have employed serial OCT imaging to evaluate the effect of statin therapy (47-53). The meta-analysis suggests that each $1 \mathrm{mg} / \mathrm{dL}$ reduction in LDL-C associates with an increase in FCT by $1.48 \mu \mathrm{m}$ during 12 -month follow-up (Figure 3). Assuming a $50 \mathrm{mg} / \mathrm{dL}$ reduction in LDL-C, the predicted mean increase $(95 \% \mathrm{CI})$ in FCT from the meta-analysis was $73.92(50.97,96.88) \mu \mathrm{m}$. For this study, the assumed treatment effect is at least $50.97 \mu \mathrm{m}$ increase, which is approximated from the lower bound $95 \%$ confidence interval. The standard deviation of change in FCT was only reported in two of the seven trials, ranging from 22 to $86 \mu \mathrm{m}$. To be conservative, the assumed common standard deviation for this study is $86 \mu \mathrm{m} .125$ completers will 
be needed to reach $90 \%$ power to detect this difference in FCT.

\section{Results}

\section{HUYGENS endpoints}

The primary endpoint of the study is the absolute change in minimum FCT in a matched arterial segment from baseline to week 50 . Secondary endpoints include the (I) percent change in minimum FCT, (II) absolute change in the average of the minimum FCT for all images and (III) absolute change in the maximum lipid arc. An additional plaque-based analysis will determine the absolute change in minimum FCT, maximum lipid arc and lipid core length in lipid rich plaques, defined as the presence of a minimum FCT $\leq 120 \mu \mathrm{m}$ and a lipid $\operatorname{arc}>90^{\circ}$ in at least three consecutive images. Measurements of plaque burden by IVUS and lipid parameters will be studied for an exploratory analysis to determine their relationship with changes in plaque phenotype on OCT imaging. Additional OCT imaging exploratory endpoints include the number of microchannels, thought to reflect plaque neovascularization, and macrophage presence and extension.

\section{Discussion}

Lipid lowering has had a major influence on cardiovascular outcomes. The use of arterial wall imaging has permitted the opportunity to characterize the effect of lipid lowering interventions on atherosclerotic plaque in vivo. HUYGENS will extend these observations to determine the impact of achieving very low LDL-C levels with a PCSK9 inhibitor on vulnerable coronary plaque features in patients with an ACS. The findings have the potential to further understand the potential link between the effects of evolocumab on the biology within the artery wall and how that may translate to a reduction in cardiovascular risk.

\section{Registration details}

This clinical trial is registered on Clinicaltrials.gov with the number NCT03570697.

\section{Acknowledgments}

The authors thank Shannon Rao, PhD of Amgen Inc., for her support.
Funding: Funding from Amgen Inc. (Thousand Oaks, CA, USA) is used to support this work, which includes the provision of editorial support.

\section{Footnote}

Reporting Checklist: The authors have completed the SPIRIT reporting checklist. Available at http://dx.doi.org/10.21037/ cdt-20-684

Conflicts of Interest: All authors have completed the ICMJE uniform disclosure form (available at http://dx.doi. org/10.21037/cdt-20-684). SJN is a recipient of a Principal Research Fellowship from the National Health and Medical Research Council of Australia and has received research support from AstraZeneca, Amgen, Anthera, CSL Behring, Cerenis, Eli Lilly, Esperion, Resverlogix, Novartis, InfraReDx and Sanofi-Regeneron and is a consultant for Amgen, Akcea, AstraZeneca, Boehringer Ingelheim, CSL Behring, Eli Lilly, Esperion, Kowa, Merck, Takeda, Pfizer, Sanofi-Regeneron and Novo Nordisk. SN reports that the Cleveland Clinic Center for Clinical Research has received funding to perform clinical trials from AbbVie, AstraZeneca, Amgen, Cerenis, Eli Lilly, Esperion, Medtronic, MyoKardia, Novartis, Pfizer, The Medicines Company, Silence Therapeutics, Takeda, and Orexigen. SN is involved in these clinical trials but receives no personal remuneration for his participation. SN consults for many pharmaceutical companies but requires them to donate all honoraria or consulting fees directly to charity so that he receives neither income nor a tax deduction. FP received consulting fees from Amgen and Abbott Vascular. SW reports research and educational grants to the institution from Abbott, Amgen, BMS, Bayer, Boston Scientific, Biotronik, Cardinal Health, CSL Behring, Daiichi Sankyo, Edwards Lifesciences, Johnson \& Johnson, Medtronic, Querbet, Polares, Sanofi, Terumo, Sinomed. YK has received research support from Kowa, and speaker honoraria from Abbott Vascular, Amgen, CSL Behring, Daiichi Sankyo, Kowa, Nipro, and Takeda. RP has received lecture fees from Amgen and Sanofi, served as a consultant for Cerenis and on advisory boards for Centerline Biomedical and Medtronic, and holds minor equity in Centerline Biomedical. TH, HK, and JL are employees of Amgen Inc. and hold Amgen stock/stock options. RS is an employee of Epirium Bio, Inc, a former employee of Amgen and identified as an inventor on at least one pending patent application relating to evolocumab. 
PJP is a recipient of a L2 Future Leader Fellowship from the National Heart Foundation of Australia (FLF102056) and a L2 Career Development Fellowship from the National Health and Medical Research Council of Australia (CDF1161506) and has received research support from Abbott Vascular, consulting fees from Amgen and Esperion and speaker honoraria from AstraZeneca, Bayer, Boehringer Ingelheim, Merck Schering-Plough and Pfizer. The other authors have no conflicts of interest to declare.

Ethical Statement: The authors are accountable for all aspects of the work in ensuring that questions related to the accuracy or integrity of any part of the work are appropriately investigated and resolved. All procedures in this study will be conducted in accordance with the Declaration of Helsinki (as revised in 2013), International Conference on Harmonization, Good Clinical Practice guidelines, and applicable regulatory requirements. The final protocol was reviewed and approved by Ethics Committees and Institutional Review Boards at each study site and by Amgen Inc. (No. RES-18-0000-283A). All study participants will provide informed consent before participating in this study.

Open Access Statement: This is an Open Access article distributed in accordance with the Creative Commons Attribution-NonCommercial-NoDerivs 4.0 International License (CC BY-NC-ND 4.0), which permits the noncommercial replication and distribution of the article with the strict proviso that no changes or edits are made and the original work is properly cited (including links to both the formal publication through the relevant DOI and the license). See: https://creativecommons.org/licenses/by-nc-nd/4.0/.

\section{References}

1. Baigent C, Blackwell L, Emberson J, et al. Efficacy and safety of more intensive lowering of LDL cholesterol: a meta-analysis of data from 170,000 participants in 26 randomised trials. Lancet 2010;376:1670-81.

2. Grundy SM, Stone NJ, Bailey AL, et al. 2018 AHA/ACC/ AACVPR/AAPA/ABC/ACPM/ADA/AGS/APhA/ASPC/ NLA/PCNA guideline on the management of blood cholesterol: a report of the American College of Cardiology/ American Heart Association Task Force on Clinical Practice Guidelines. Circulation 2019;139:e1082-143.

3. Jones PH, Nair R, Thakker KM. Prevalence of dyslipidemia and lipid goal attainment in statin-treated subjects from 3 data sources: a retrospective analysis. J Am Heart Assoc 2012;1:e001800.

4. Libby P. The forgotten majority: unfinished business in cardiovascular risk reduction. J Am Coll Cardiol 2005;46:1225-8.

5. Banach M, Rizzo M, Toth PP, et al. Statin intolerance - an attempt at a unified definition. Position paper from an International Lipid Expert Panel. Arch Med Sci 2015;11:1-23.

6. Ballantyne CM, Houri J, Notarbartolo A, et al. Effect of ezetimibe coadministered with atorvastatin in 628 patients with primary hypercholesterolemia: a prospective, randomized, double-blind trial. Circulation 2003;107:2409-15.

7. Cannon CP, Blazing MA, Giugliano RP, et al. Ezetimibe added to statin therapy after acute coronary syndromes. $\mathrm{N}$ Engl J Med 2015;372:2387-97.

8. Baigent C, Landray MJ, Reith C, et al. The effects of lowering LDL cholesterol with simvastatin plus ezetimibe in patients with chronic kidney disease (Study of Heart and Renal Protection): a randomised placebo-controlled trial. Lancet 2011;377:2181-92.

9. Pinkosky SL, Filippov S, Srivastava RA, et al. AMPactivated protein kinase and ATP-citrate lyase are two distinct molecular targets for ETC-1002, a novel small molecule regulator of lipid and carbohydrate metabolism. J Lipid Res 2013;54:134-51.

10. Cramer CT, Goetz B, Hopson KL, et al. Effects of a novel dual lipid synthesis inhibitor and its potential utility in treating dyslipidemia and metabolic syndrome. J Lipid Res 2004;45:1289-301.

11. Ballantyne CM, Davidson MH, Macdougall DE, et al. Efficacy and safety of a novel dual modulator of adenosine triphosphate-citrate lyase and adenosine monophosphate-activated protein kinase in patients with hypercholesterolemia: results of a multicenter, randomized, double-blind, placebo-controlled, parallelgroup trial. J Am Coll Cardiol 2013;62:1154-62.

12. Goldberg AC, Leiter LA, Stroes ESG, et al. Effect of bempedoic acid vs placebo added to maximally tolerated statins on low-density lipoprotein cholesterol in patients at high risk for cardiovascular disease: the CLEAR wisdom randomized clinical trial. JAMA 2019;322:1780-8.

13. Abifadel M, Varret M, Rabes JP, et al. Mutations in PCSK9 cause autosomal dominant hypercholesterolemia. Nat Genet 2003;34:154-6.

14. Cohen J, Pertsemlidis A, Kotowski IK, et al. Low LDL cholesterol in individuals of African descent resulting 
from frequent nonsense mutations in PCSK9. Nat Genet 2005;37:161-5.

15. Cohen JC, Boerwinkle E, Mosley THJ, et al. Sequence variations in PCSK9, low LDL, and protection against coronary heart disease. N Engl J Med 2006;354:1264-72.

16. Maxwell KN, Breslow JL. Adenoviral-mediated expression of Pcsk9 in mice results in a low-density lipoprotein receptor knockout phenotype. Proc Natl Acad Sci U S A 2004;101:7100-5.

17. Seidah NG, Benjannet S, Wickham L, et al. The secretory proprotein convertase neural apoptosisregulated convertase 1 (NARC-1): liver regeneration and neuronal differentiation. Proc Natl Acad Sci U S A 2003;100:928-33.

18. Robinson JG, Nedergaard BS, Rogers WJ, et al. Effect of evolocumab or ezetimibe added to moderate- or highintensity statin therapy on LDL-C lowering in patients with hypercholesterolemia: the LAPLACE-2 randomized clinical trial. JAMA 2014;311:1870-82.

19. Blom DJ, Hala T, Bolognese M, et al. A 52-week placebocontrolled trial of evolocumab in hyperlipidemia. N Engl J Med 2014;370:1809-19.

20. Robinson JG, Farnier M, Kastelein JJP, et al. Relationship between alirocumab, PCSK9, and LDL-C levels in four phase 3 ODYSSEY trials using 75 and $150 \mathrm{mg}$ doses. J Clin Lipidol 2019;13:979-88.e10.

21. Santos RD, Stein EA, Hovingh GK, et al. Long-term evolocumab in patients with familial hypercholesterolemia. J Am Coll Cardiol 2020;75:565-74.

22. Nissen SE, Stroes E, Dent-Acosta RE, et al. Efficacy and tolerability of evolocumab vs ezetimibe in patients with muscle-related statin intolerance: the GAUSS-3 randomized clinical trial. JAMA 2016;315:1580-90.

23. Sabatine MS, Giugliano RP, Keech AC, et al. Evolocumab and clinical outcomes in patients with cardiovascular disease. N Engl J Med 2017;376:1713-22.

24. Schwartz GG, Steg PG, Szarek M, et al. Alirocumab and cardiovascular outcomes after acute coronary syndrome. N Engl J Med 2018;379:2097-107.

25. Ballantyne CM. Clinical trial endpoints: angiograms, events, and plaque instability. Am J Cardiol 1998;82:5M-11M.

26. Ballantyne CM, Raichlen JS, Nicholls SJ, et al. Effect of rosuvastatin therapy on coronary artery stenoses assessed by quantitative coronary angiography: a study to evaluate the effect of rosuvastatin on intravascular ultrasound-derived coronary atheroma burden. Circulation 2008;117:2458-66.
27. Smilde TJ, van Wissen $S$, Wollersheim $H$, et al. Effect of aggressive versus conventional lipid lowering on atherosclerosis progression in familial hypercholesterolaemia (ASAP): a prospective, randomised, double-blind trial. Lancet 2001;357:577-81.

28. Taylor AJ, Kent SM, Flaherty PJ, et al. ARBITER: Arterial biology for the investigation of the treatment effects of reducing cholesterol: a randomized trial comparing the effects of atorvastatin and pravastatin on carotid intima medial thickness. Circulation 2002;106:2055-60.

29. Nissen SE, Tuzcu EM, Schoenhagen P, et al. Effect of intensive compared with moderate lipid-lowering therapy on progression of coronary atherosclerosis: a randomized controlled trial. JAMA 2004;291:1071-80.

30. Nissen SE, Nicholls SJ, Sipahi I, et al. Effect of very high-intensity statin therapy on regression of coronary atherosclerosis: the ASTEROID trial. JAMA 2006;295:1556-65.

31. Nicholls SJ, Ballantyne CM, Barter PJ, et al. Effect of two intensive statin regimens on progression of coronary disease. N Engl J Med 2011;365:2078-87.

32. Nissen SE, Tuzcu EM, Schoenhagen P, et al. Statin therapy, LDL cholesterol, C-reactive protein, and coronary artery disease. N Engl J Med 2005;352:29-38.

33. Puri R, Nissen SE, Libby P, et al. C-reactive protein, but not low-density lipoprotein cholesterol levels, associate with coronary atheroma regression and cardiovascular events after maximally intensive statin therapy. Circulation 2013;128:2395-403.

34. Crisby M, Nordin-Fredriksson G, Shah PK, et al. Pravastatin treatment increases collagen content and decreases lipid content, inflammation, metalloproteinases, and cell death in human carotid plaques: implications for plaque stabilization. Circulation 2001;103:926-33.

35. Puri R, Nicholls SJ, Shao M, et al. Impact of statins on serial coronary calcification during atheroma progression and regression. J Am Coll Cardiol 2015;65:1273-82.

36. Kini AS, Baber U, Kovacic JC, et al. Changes in plaque lipid content after short-term intensive versus standard statin therapy: the YELLOW trial (reduction in yellow plaque by aggressive lipid-lowering therapy). J Am Coll Cardiol 2013;62:21-9.

37. Underhill HR, Yuan C, Zhao XQ, et al. Effect of rosuvastatin therapy on carotid plaque morphology and composition in moderately hypercholesterolemic patients: a high-resolution magnetic resonance imaging trial. Am Heart J 2008;155:584.e1-8.

38. Tahara N, Kai H, Ishibashi M, et al. Simvastatin attenuates 
plaque inflammation: evaluation by fluorodeoxyglucose positron emission tomography. J Am Coll Cardiol 2006;48:1825-31.

39. Kataoka Y, Puri R, Hammadah M, et al. Cholesterol crystals associate with coronary plaque vulnerability in vivo. J Am Coll Cardiol 2015;65:630-2.

40. Prati F, Romagnoli E, Gatto L, et al. Relationship between coronary plaque morphology of the left anterior descending artery and 12 months clinical outcome: the CLIMA study. Eur Heart J 2020;41:383-91.

41. Kataoka Y, Puri R, Hammadah M, et al. Spotty calcification and plaque vulnerability in vivo: frequencydomain optical coherence tomography analysis. Cardiovasc Diagn Ther 2014;4:460-9.

42. Tearney GJ, Regar E, Akasaka T, et al. Consensus standards for acquisition, measurement, and reporting of intravascular optical coherence tomography studies: a report from the International Working Group for Intravascular Optical Coherence Tomography Standardization and Validation. J Am Coll Cardiol 2012;59:1058-72.

43. Barlis P, Serruys PW, Gonzalo N, et al. Assessment of culprit and remote coronary narrowings using optical coherence tomography with long-term outcomes. Am J Cardiol 2008;102:391-5.

44. Tanaka A, Imanishi T, Kitabata H, et al. Distribution and frequency of thin-capped fibroatheromas and ruptured plaques in the entire culprit coronary artery in patients with acute coronary syndrome as determined by optical coherence tomography. Am J Cardiol 2008;102:975-9.

45. Xing L, Higuma T, Wang Z, et al. Clinical significance of lipid-rich plaque detected by optical coherence tomography: a 4-year follow-up study. J Am Coll Cardiol 2017;69:2502-13.

46. Kataoka Y, Hammadah M, Puri R, et al. Plaque microstructures in patients with coronary artery disease who achieved very low low-density lipoprotein cholesterol levels. Atherosclerosis 2015;242:490-5.

47. Kataoka Y, Puri R, Hammadah M, et al. Frequencydomain optical coherence tomographic analysis of plaque microstructures at nonculprit narrowings in patients receiving potent statin therapy. Am J Cardiol 2014;114:549-54.

48. Dai J, Hou J, Xing L, et al. Is age an important factor for vascular response to statin therapy? A serial optical coherence tomography and intravascular ultrasound study. Coron Artery Dis 2017;28:209-17.
49. Hattori K, Ozaki Y, Ismail TF, et al. Impact of statin therapy on plaque characteristics as assessed by serial OCT, grayscale and integrated backscatter-IVUS. JACC Cardiovasc Imaging 2012;5:169-77.

50. Hou J, Xing L, Jia H, et al. Comparison of intensive versus moderate lipid-lowering therapy on fibrous cap and atheroma volume of coronary lipid-rich plaque using serial optical coherence tomography and intravascular ultrasound imaging. Am J Cardiol 2016;117:800-6.

51. Komukai K, Kubo T, Kitabata H, et al. Effect of atorvastatin therapy on fibrous cap thickness in coronary atherosclerotic plaque as assessed by optical coherence tomography: the EASY-FIT study. J Am Coll Cardiol 2014;64:2207-17.

52. Nishio R, Shinke T, Otake H, et al. Stabilizing effect of combined eicosapentaenoic acid and statin therapy on coronary thin-cap fibroatheroma. Atherosclerosis 2014;234:114-9.

53. Takarada S, Imanishi T, Kubo T, et al. Effect of statin therapy on coronary fibrous-cap thickness in patients with acute coronary syndrome: assessment by optical coherence tomography study. Atherosclerosis 2009;202:491-7.

54. Nicholls SJ, Puri R, Anderson T, et al. Effect of evolocumab on progression of coronary disease in statintreated patients: the GLAGOV randomized clinical trial. JAMA 2016;316:2373-84.

55. Nicholls SJ, Puri R, Anderson T, et al. Effect of evolocumab on coronary plaque composition. J Am Coll Cardiol 2018;72:2012-21.

56. Stiekema LCA, Stroes ESG, Verweij SL, et al. Persistent arterial wall inflammation in patients with elevated lipoprotein(a) despite strong low-density lipoprotein cholesterol reduction by proprotein convertase subtilisin/kexin type 9 antibody treatment. Eur Heart J 2019;40:2775-81.

Cite this article as: Nicholls SJ, Nissen SE, Prati F, Windecker S, Kataoka Y, Puri R, Hucko T, Kassahun H, Liao J, Somaratne R, Butters J, Di Giovanni G, Jones S, Psaltis PJ. Assessing the impact of PCSK9 inhibition on coronary plaque phenotype with optical coherence tomography: rationale and design of the randomized, placebo-controlled HUYGENS study. Cardiovasc Diagn Ther 2021;11(1):120-129. doi: $10.21037 / \mathrm{cdt}-20-684$ 